\title{
La question du réalisme scientifique : un problème épistémologique central
}

\section{Angèle Kremer Marietti}

\section{(2) OpenEdition}

\section{Journals}

Édition électronique

URL : http://journals.openedition.org/ress/575

DOI : $10.4000 /$ ress.575

ISSN : 1663-4446

Éditeur

Librairie Droz

Édition imprimée

Date de publication : 1 août 2002

Pagination : $59-83$

ISBN : 2-600-00806-3

ISSN : 0048-8046

Référence électronique

Angèle Kremer Marietti, « La question du réalisme scientifique : un problème épistémologique central », Revue européenne des sciences sociales [En ligne], XL-124 | 2002, mis en ligne le 01 décembre 2009, consulté le 19 avril 2019. URL : http://journals.openedition.org/ress/575 ; DOI : 10.4000/ress.575 


\section{LA QUESTION DU RÉALISME SCIENTIFIQUE: UN PROBLÈME ÉPISTÉMOLOGIQUE CENTRAL}

Je traite le problème du réalisme en commençant par le poser historiquement dans l'horizon du tournant du $\mathrm{XIX}^{\mathrm{e}}$ au $\mathrm{XX}^{\mathrm{e}}$ siècle, du point de vue de son observation par Werner Heisenberg et de sa solution par Gaston Bachelard dont l'épistémologie surplombe les premières et grandes révolutions qui furent celles du $\mathrm{XX}^{\mathrm{e}}$ siècle. Ensuite, $\mathrm{j}$ 'étudie comment Bachelard a procédé à une éducation du nouvel esprit scientifique qu'il ouvre à l'inférence de la Réalité cachée. Après quoi je reviens, pour ainsi dire, au point de départ en envisageant le réalisme et l'anti-réalisme d'une manière générale, pour dissiper ce que j'appelle le faux dualisme observable/inobservable et me poser délibérément contre le «réalisme immédiat» de la perception ordinaire. Je termine en examinant un paradoxe de la mécanique quantique et sa solution.

I. Position historique du problème

II. L'éducation du nouvel esprit scientifique ouvert à l'inférence de la Réalité cachée

III. Du réalisme et de l'anti-réalisme en général

IV. Le faux dualisme observable/inobservable

V. Contre le réalisme immédiat de la perception ordinaire

VI. La mesure des grandeurs en physique quantique

VII. Conclusion.

\section{POSITION HISTORIQUE DU PROBLÈME}

G. Bachelard (1884-1962) avait vu juste quand il écrivait en 1934: «Tôt ou tard, c'est la pensée scientifique qui deviendra le thème fondamental de la polémique philosophique; cette pensée conduira à substituer aux métaphysiques intuitives et immédiates les métaphysiques discursives objectivement rectifiées.» ${ }^{1}$

Il semble que Bachelard ait résumé par avance un aspect fondamental de la situation épistémologique présente, puisque la polémique philosophique actuelle porte en effet surtout sur la pensée scientifique à laquelle on voudrait contester la réalité de ses objets; de plus, il semble bien que la raison de cette contestation en soit, comme le suggérait Bachelard, la substitution aux anciennes «métaphysiques intuitives et immédiates » d'autres processus d'approche autant discursifs qu'ob-

\footnotetext{
Le nouvel esprit scientifique, Paris, PUF, 1946 (1934), p. 2.
} 
jectivement rectifiés, c'est-à-dire reposant sur une objectivité non plus immédiate mais fondée sur certains calculs très précis. La polémique épistémologique actuelle dépend certainement de plusieurs circonstances, parmi lesquelles une tendance vers le relativisme épistémologique dont fait état la pensée postmoderne ${ }^{2}$; mais l'une d'entre elles, plus ancienne, peut certainement être aussi la méfiance envers ces discours dits «objectivement rectifiés» parce qu'ils sont reliés à leurs objets d'une manière indirecte, d'où la critique contemporaine concernant leur réalité.

Il est vrai qu'au tournant du $\mathrm{XIX}^{\mathrm{e}}$ au $\mathrm{XX}^{\mathrm{e}}$ siècle, ce qui distinguait les partis épistémologiques, c'était l'opposition entre le réalisme et le rationalisme: le développement des mathématiques et leur succès dans l'élaboration de la science physique faisaient suspecter la réalité des objets qu'elles traitaient. Autrement dit, le rationalisme mathématique rendait suspect le réalisme scientifique. Du moins, les deux attitudes se retrouvaient pour ainsi dire confrontées mais nécessairement complémentaires dans l'élaboration de la science. Le matérialisme du XIX siècle faisait une image simpliste de l'univers, selon la version éclairée qu'en donne W. Heisenberg ${ }^{3}$ (1901-1976): les atomes constituaient un étant inaltérable; ils se mouvaient dans l'espace et dans le temps en provoquant par leur disposition et leurs mouvements réciproques les phénomènes variés de notre univers sensible. Heisenberg ajoutait que même si, dès la deuxième moitié du siècle, cette image avait été quelque peu ébranlée par la science de l'électricité pour laquelle le réel proprement dit n'était plus la matière mais le champ électrique, on pouvait continuer à maintenir, par le détour d'un éther matériel supportant ces champs électriques, la vision matérialiste de la physique de l'atome. On voulait croire que les modifications des champs électriques étaient des processus se déroulant dans l'espace et dans le temps; et l'on pouvait encore imaginer que les champs électriques étaient provoqués par les atomes: on pouvait même les utiliser pour expliquer le mouvement des atomes. Et, d'ailleurs, lorsque, plus tard, on aura découvert les particules élémentaires telles que les protons, les neutrons et les électrons, on persistera à croire dans une réalité objective fondamentale, sans qu'il soit fait mention des processus d'observation. Mais on remarquait déjà, comme H. Bergson ${ }^{4}$ (18591941) dans L'évolution créatrice, en 1907, qu'il devenait impossible de se représenter les propriétés, par exemple de l'éther dont on parlait à l'époque, ou même de l'électricité: "Plus la physique progresse, plus elle montre l'impossibilité de se représenter les propriétés de l'éther ou de l'électricité, base probable de tous les corps, sur le modèle des propriétés de la matière que nous apercevons.»

Toutefois, il faut pourtant ajouter que des transformations profondes ${ }^{5}$ par rapport à cette vue mécanistique de la nature étaient déjà intervenues; il faut noter, en

Cf. J. Bricmont, Postmodernism and its problems with science, conférence donnée en décembre 1996 sur l'invitation de la Société Finnoise de Mathématiques; voir sur le site DOGMA: http://dogma.free.fr

3 W. Heisenberg, La nature dans la physique contemporaine, traduit de l'allemand par U. Karvelis \& A. E. Leroy, Paris, Gallimard, 1962 (éd. originale 1955); nouvelle édition introduite par C. Chevalley, Paris, Gallimard, Collection Folio-Essais, 2000.

4 Cf. H. Bergson, Euvres, texte annoté par A. Robinet, introduit par H. Gouhier, Paris, PUF, 1970 (1959); voir L'évolution créatrice, in Euvres, p. 803.

5 A. Kremer-Marietti, Philosophie des sciences de la nature, Paris, PUF, 1999, chap. V, pp. 181257, Philosophies scientifiques du XX $X^{e}$ siècle. 
effet, un certain nombre de nouveautés dans le champ des sciences de la nature: dès 1892, H. A. Lorentz (1853-1928) avait commencé la publication d'une série d'articles sur la théorie de l'électron, une particule élémentaire chargée négativement dont J. J. Thomson (1856-1940) prouvera l'existence en 1897; de plus, en 1895, W. C. Röntgen (1845-1923) avait découvert les rayons X (ou les ondes électromagnétiques). Quelques mois plus tard, en 1896, H. Becquerel (1852-1908) vit dans l'uranium une source de radioactivité, c'est-à-dire l'émission spontanée de particules subatomiques et de radiations électromagnétiques à haute fréquence; après quoi, P. Curie (1859-1906) et M. Curie (1867-1934) découvraient en 1898 la radioactivité du radium et du polonium. En 1900 - alors qu'A. Einstein (18791955) terminait ses études à l'École polytechnique de Zurich -, M. Planck (18581947) analysait l'équilibre thermique entre matière et rayonnement et découvrait ce qu'il appelait «le quantum d'action élémentaire», en n'usant d'aucune théorie mécanique concernant la théorie de la chaleur. L'hypothèse de Planck était une hypothèse qu'on dit ad hoc, mais sa découverte exprimait quelque chose qu'il était impossible d'énoncer dans l'ancienne mécanique: une équivalence entre énergie et fréquence. En 1905, ce sera l'année miraculeuse, durant laquelle Einstein publiera cinq articles à la base des deux premières révolutions du $\mathrm{XX}^{\mathrm{e}}$ siècle: celle de la Relativité et celle des quanta ${ }^{6}$.

L. Boltzmann (1844-1906) créa la mécanique statistique, qui modèle un système dans les termes du comportement moyen du grand nombre d'atomes et de molécules formant le système: les lois de base de cette théorie découlent de la théorie newtonienne; on y examine les conséquences de la connaissance incomplète d'un système mécanique complexe, aussi le principe du déterminisme y estil maintenu. A la même époque, J. C. Maxwell (1831-1879) et Boltzmann créèrent la théorie cinétique des gaz dans laquelle une description statistique des mouvements moléculaires permet d'expliquer toutes les grandeurs caractéristiques des gaz (pression, température, énergie, etc). Quant à Boltzmann, on peut le considérer comme un pionnier de la mécanique quantique pour deux raisons. Son interprétation statistique de la seconde loi de la thermodynamique permit d'introduire la Théorie des probabilités dans une loi fondamentale de physique: voilà qui donnait l'occasion de contredire le principe classique du déterminisme. Alors que depuis 1897 Boltzmann l'invitait à utiliser ses méthodes statistiques pour le traitement de la radiation du corps noir, Max Planck attendit 1900 avant d'admettre les méthodes statistiques de Boltzmann. Autre progrès de Boltzmann vers la mécanique quantique: une méthode qu'il utilisait déjà en 1872, l'introduction des niveaux d'énergie discrète.

\section{L'ÉDUCATION DU NOUVEL ESPRIT SCIENTIFIQUE OUVERT À L'INFÉRENCE DE LA RÉALITÉ CACHÉE}

Dans son ouvrage de 1934, Le nouvel esprit scientifique, Bachelard en appelait très justement au «double sens de la preuve scientifique qui s'affirme dans

$6 \quad$ Philosophie des sciences de la nature, pp. 187-201. 
l'expérience aussi bien que dans le raisonnement, à la fois dans un contact avec la réalité et dans une référence à la raison ${ }^{7}$. Il y expose clairement la situation épistémologique qui s'est confirmée au tournant du $\mathrm{XX}^{\mathrm{e}}$ siècle, à propos de laquelle il cherche à expliquer «la réalisation du rationnel dans l'expérience physique ${ }^{8}$. C'est pourquoi il proposait à l'épistémologue de se placer «à la croisée des chemins $[\ldots]$ entre le réalisme et le rationalisme» ${ }^{9}$.

Il semble utile de rappeler comment Bachelard résout le problème qu'il voit posé à ce moment-là; au scepticisme des mathématiciens conventionalistes de son époque, il oppose l'exigence que soit prise en considération la dialectique des pensées géométriques; il rappelle comment les euclidiens avaient refusé la «géométrie généralisée» de N. I. Lobatchewsky ${ }^{10}$ (1792-1856). Aussi Bachelard demande-t-il: «Qu'est-ce que la croyance à la réalité?» ${ }^{11}$ Et il répond: «c'est la conviction que l'on retrouvera plus dans le réel caché que dans le donné évident.» ${ }^{12}$ Bachelard s'efforce ainsi de faire comprendre comment l'astronomie newtonienne est un cas particulier de la «Panastronomie d'Einstein», de même comment la géométrie euclidienne est un cas particulier de la «Pangéométrie de Lobatchewsky ${ }^{13}$; et il fait remarquer: tandis que «la Géométrie de Lobatchewsky a attaqué la primitivité de l'idée de parallélisme», la théorie de la Relativité attaque «la primitivité de l'idée de simultanéité ${ }^{14}$.

On voit que Bachelard s'est donné essentiellement une tâche didactique, celle de faire admettre à une génération nouvelle les changements importants survenus dans les repères intellectuels de la pensée scientifique. La position de Bachelard, même si elle est - et à juste titre - réaliste-rationaliste, n'en est pas moins foncièrement réaliste, en ce sens qu'il voit opérer dans les nouveaux processus de la pensée scientifique des systèmes d'équivalences ou de correspondances qui habituent l'esprit à une nouvelle conception de la réalité et lui donnent une sécurité philosophique que l'on chercherait en vain par exemple, dans la philosophie de Heisenberg $^{15}$, puisqu'il voit, confirmée d'ailleurs autant chez Einstein que chez Heisenberg, «une sorte de doublement expérimental des notions rationnelles ${ }^{16}$ qui ne sont plus absolues mais relatives à une expérience précise.

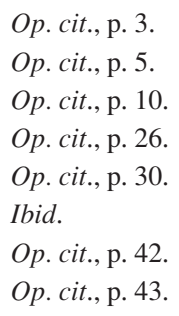

Je renvoie à la très pénétrante analyse développée par C. Chevalley dans l'Introduction qu'elle a apportée à l'édition du recueil de Heisenberg, La nature dans la physique contemporaine, et, en particulier à la conférence de 1953 qui ouvre le volume, et qui constitue un aboutissement de la philosophie de Heisenberg, déjà exprimée en 1941 et 1942, publiée à titre posthume et sans titre par H. Rechenberg dans W. Heisenberg, Gesammelte Werke, Berlin, Springer, 1984, 1985, 1989 et 1993 ; voir aussi Munich, Piper, 1984, 1985, 1986 et 1989. Cf. W. Heisenberg, La nature dans la physique contemporaine, Paris, Gallimard, Collection Folio-Essais, 2000. Voir, entre autres, page 11, la note 2 de C. Chevalley. Cf W. Heisenberg, Philosophie. Le manuscrit de 1942, Paris, Le Seuil, 1998. 
Là où Bachelard reconnaît la réalité de l'événement ou du comportement des nouveaux objets scientifiques, ce n'est pas pour déplorer comme le fait Heisenberg la perte de la «nature 'en soi'» ${ }^{17}$ ou la «perte du sol de l'expérience immédiate ${ }^{18}$. Car la réalité en soi n'est plus dans le discours bachelardien que le fait d'un réalisme immédiat, position désormais à dépasser. Pour Bachelard, il est clair que l'homo faber a laissé définitivement place à l' homo mathematicus ${ }^{19}$ : on est bien passé de l'âge d'un Réel solide à celui d'une «Réalité cachée ». À l'époque, c'est le Calcul tensoriel qui, selon la parole de Langevin «sait mieux la physique que le Physicien lui-même $»^{20}$. Et Bachelard souligne la «force inductive et inventive que l'esprit acquiert en maniant le Calcul tensoriel ${ }^{21}$. Toujours, en distançant l' «expérience immédiate ${ }^{22}$ ou l' «expérience présente ${ }^{23}$, le nouvel esprit scientifique «pense toutes les possibilités expérimentales ${ }^{24}$ : là, je ne peux faire autrement que de rappeler une notion importante de la théorie kantienne de la connaissance - que l'on néglige le plus souvent - et qui est la notion de «l'expérience possible». Pourtant, Bachelard souligne combien Heisenberg, qui d'un point de vue positiviste insistait pour que toutes les notions aient un sens expérimental, acceptait lui-même de s'appuyer sur des «expériences fictives » ${ }^{25}$; c'est pourquoi Bachelard conclut dans l'esprit kantien: «Il suffit qu'elles soient possibles ${ }^{26}$. De la sorte, Bachelard intervenait aussi dans la polémique entre Hans Reichenbach et Oskar Kraus ${ }^{27}$, justement en pensant les possibilités expérimentales comme caractérisant assez la physique mathématique pour que le possible s'y rapprochât du réel et s'éloignât en effet de ce que Bachelard appelait les «traductions plus ou moins fantaisistes de la philosophie du comme $s i{ }^{28}$.

Bachelard combat donc un matérialisme « concret» qui mutile en fait la notion de matière en la limitant et en la localisant dans un espace réduit; sa vision se rapproche alors de celle que fera Heisenberg qui, dans sa conférence de 1953, décrira le matérialisme étroit du XIX ${ }^{\mathrm{e}}$ siècle. Mais, n'abandonnant pas l'idéal de la totalité, Bachelard imagine «cette immense architecture réaliste [qui] fut touchée par une mathématique complexe et subtile $»^{29}$, là où Heisenberg pensera comme vaine la possibilité d'une conception ou d'une science d'une totalité du Réel. C'est pourquoi Bachelard a entrepris un travail de préparation et même d'éducation des esprits à ce changement nécessaire relativement aux images spatio-temporelles

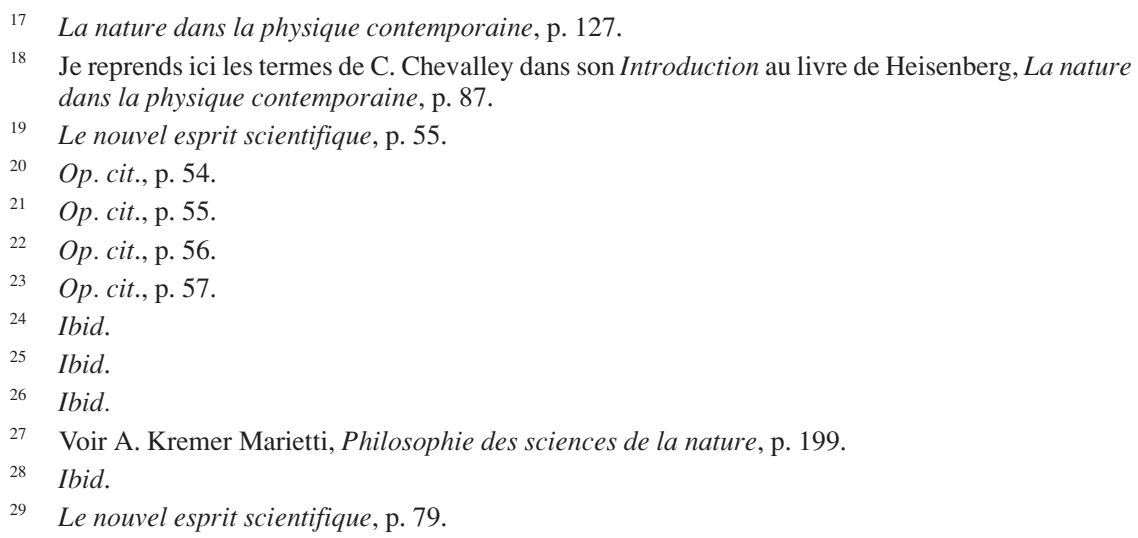


qui jusque-là avaient servi à décrire les phénomènes naturels et qu'il fallait abandonner quand il s'agissait désormais des processus atomiques. Avec la mécanique quantique, l'ère de la géométrie intuitive avait fait long feu. Et, comme le rappelle clairement Catherine Chevalley ${ }^{30}$ en suivant N. Bohr ${ }^{31}$ (1885-1962), ce qui avait changé, c'étaient: 1) la définition de l'objectivation; 2) la question du langage des théories; 3) la division sujet/objet.

Certes, les concepts classiques étaient dépassés. De ce fait, Heisenberg, dès $1927^{32}$, avait jugé nécessaire de repenser la théorie kantienne de la connaissance, du moins telle qu'elle avait été assimilée par les exégètes de I. Kant (1724-1804). Sans renoncer à Kant, Bachelard veut «ruiner le réalisme naïf ${ }^{33}$ et il propose maintenant de parler, en ce qui concerne photon, électron ou atome, «plutôt de réalisation que de réalité ${ }^{34}$. D'autant plus que le réalisme naïf «voudrait former partout des choses aux caractères permanents $\gg^{35}$. Ainsi, renversant la tendance classique devenue triviale, Bachelard va même jusqu'à exiger de transmuter les « valeurs réalistes » et jusqu'à déclarer que les espaces de configuration, opposés à l'espace intuitif, sont, du point de vue de la pensée synthétique, «plus réels que l'espace ordinaire ${ }^{36}$. Il écrit en termes kantiens : «On peut les considérer comme de véritables formes a priori de la schématisation ${ }^{37}$; ce sont en tout cas, écrit-il, des «espaces quasi naturels pour les études de la probabilité ${ }^{38}$ qui impliquent des éléments très nombreux et des espaces aux dimensions riches. Bachelard insistera pour distinguer le probable du fait de la simple ignorance ou même de l'irréel.

Bachelard met en évidence que la physique indéterministe de Heisenberg «absorbe» la physique déterministe et, au lieu de parler de l'absence de division de l'objet et du sujet, il parlera plutôt de «l'interférence essentielle de la méthode et de l'objet» ou de «l'action des procédés de la méthode sur l'objet observé ${ }^{39}$. Pour lui, le réaliste immédiat doit «passer de la géométrisation intuitive à l'arithmétisation discursive et revenir vers une définition probabilitaire des champs ${ }^{40}$.

Sensible lui-même à ce qu'il appelle «le trouble de la désignation objective ${ }^{41}$, Bachelard, en matière d'atomes, proposera de considérer que nous communiquons avec un groupe, et qu'il faut alors parler d'une «réalité collective ${ }^{42}$. Si bien que lorsque Bachelard parle d' «entraînement réaliste», il veut dire un

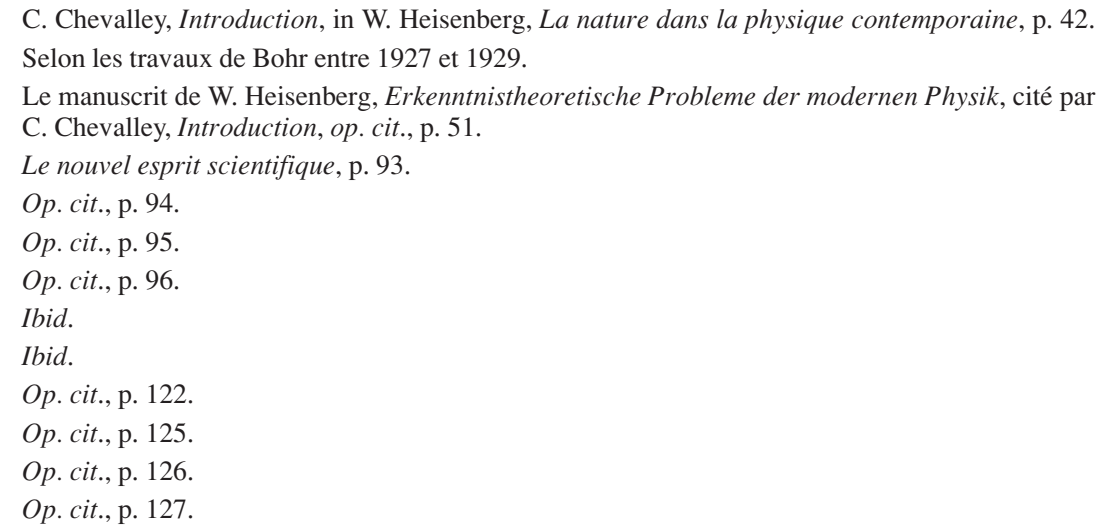


entraînement au «réalisme élémentaire» ${ }^{43}$; et il assimile les réactions qu'il constate autour de lui devant l'infiniment petit physique aux réactions embarrassées du XVII ${ }^{e}$ siècle devant l' infiniment petit mathématique.

Évoquant les questions de langage, Bachelard demande à propos du terme spin si le physicien vise une rotation réelle; et il répond que le spin est moins imaginé que pensé; de plus, quand nous imaginons nous nous fondons sur nos sensations, tandis que lorsque nous pensons ce ne peut être que mathématiquement. Si, dans les préparatifs expérimentaux, le physicien part du réel du sens commun, il fait volte-face dès qu'intervient la pensée expérimentale effective ${ }^{44}$. Or, pour Bachelard, c'est précisément entre la construction d'une nouvelle réalité et l'évanouissement d'un objet scientifique que se situe la pensée non réaliste ou anti-réaliste, c'est-à-dire au moment où s'infléchit la pensée scientifique avant les «périodes de science acquise, assise, expliquée, enseignée ${ }^{45}$, c'est-à-dire avant les périodes de ce que T.S. Kuhn (1922-1996) appellera la «science normale ${ }^{46}$.

Aussi le réalisme est-il vu par Bachelard comme devant être constamment reclassé, du fait qu'il y a ce que Bachelard appelle aussi des «révolutions fructueuses de la pensée scientifique ${ }^{47}$. Le fait est que, dans la microphysique, les bases du réel ne doivent plus être découvertes, mais inférées ${ }^{48}$. Bachelard confirme cette «conscience de la totalité » ${ }^{49}$ qu'il perçoit donc dans la pensée de la Physique mathématique qui offre « des axes chaque jour plus nombreux à l'objectivation scientifique $»^{50}$.

Donnant l'exemple de la goutte de cire du physicien contemporain, mis en parallèle avec l'exemple du morceau de cire cartésien, Bachelard démontre l'objectivation progressive à l'œuvre: la cire purifiée par l'expérience factice représente un moment précis de la méthode d'objectivation ${ }^{51}$; le physicien fait fondre le fragment de cire, puis le fait solidifier: sur plus de quatre pages, Bachelard expose les techniques, les hypothèses et les constructions mathématiques qui s'additionnent dans les expériences sur la goutte de cire pour aboutir à la constatation suivante: «Les qualités du réel scientifique sont ainsi, au premier chef, des fonctions de nos méthodes rationnelles $»^{52}$.

Tout au long de son livre d'instruction du nouvel esprit scientifique, Bachelard insiste donc sur l'accompagnement psychologique de son lecteur attentif, tout en répondant, au passage, à maintes polémiques de son époque: au moins à celle de l'objectivation, à celle du langage et à celle de la division sujet/objet, qui ont lésé la conscience classique du fait des révolutions scientifiques du $\mathrm{XX}^{\mathrm{e}}$ siècle.

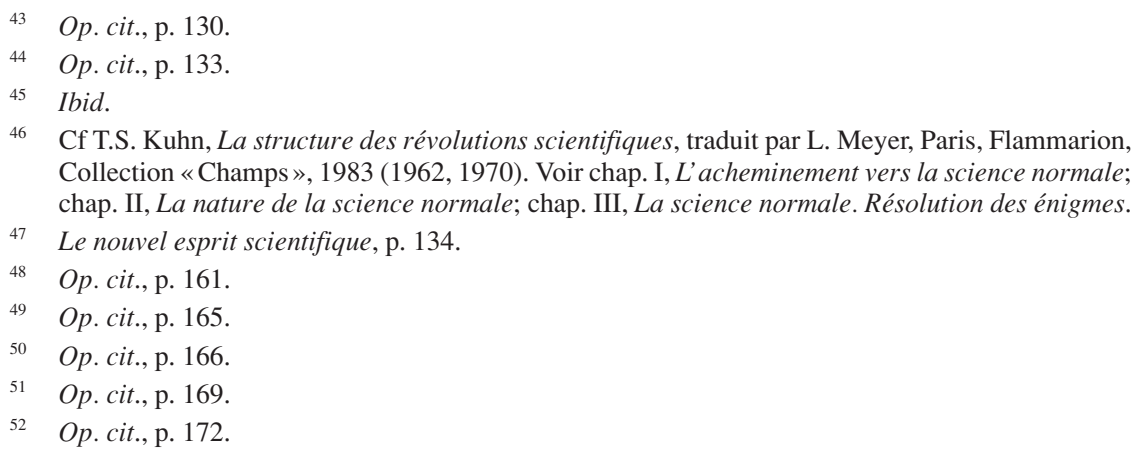




\section{DU RÉALISME ET DE L'ANTI-RÉALISME EN GÉNÉRAL}

Mis à part les sceptiques qui nient depuis toujours que nous ayons la faculté ou le droit d'affirmer la réalité de l'objet décrit dans quelque énoncé que ce soit, il est vrai que les développements de la microphysique, en nécessitant des processus indirects, ont favorisé plusieurs types de positions anti-réalistes. Ces développements ont fait douter les meilleurs scientifiques du $\mathrm{XIX}^{\mathrm{e}}$ et du $\mathrm{XX}^{\mathrm{e}}$ siècles de la réalité des objets scientifiques.

D’une manière générale, nous devons surtout prendre en considération la position des constructionnistes qui suspectent notre capacité d'atteindre la vérité à propos d'un objet de pensée s'il n'est pas «directement observable». Je discuterai directement cet argument majeur en tenant compte de la position des instrumentalistes qui nient qu'il y ait des «conditions de vérité» relatives aux énoncés perceptuels et même conceptuels sur les objets du monde. Mais s'il est vrai que la question des «conditions de vérité» implique depuis toujours, depuis Spinoza (1632-1677) et Kant, des décisions régulatrices en ce qui concerne les modes de l'observation et de l'expérimentation, il n'en demeure pas moins que, ces précautions étant prises, il reste de fortes chances pour que les énoncés scientifiques portent sur des objets nantis de réalité. Quant aux positions idéalistes et conceptualistes, elles affirmeraient douter de l'indépendance de la réalité des objets scientifiques pour penser au contraire qu'ils dépendent entièrement de notre facture d'esprit - en particulier, soit du langage, soit de schèmes conceptuels que nous leur imposerions (on a attribué abusivement à Kant une telle position). Certes, notre constitution biologique et intellectuelle entre indéniablement en jeu dans l'activité symbolisatrice en général et dans celle de la science en particulier, mais ce n'est pas une raison suffisante pour conclure que la réalité des objets scientifiques dépend intégralement de notre facture d'esprit, que ce soit de notre langage ou de schèmes conceptuels a priori.

Au XIX ${ }^{\mathrm{e}}$ siècle, les phénoménalistes ont, avec le physicien E. Mach (18381916), douté de l'existence des objets dits «non observables»; au milieu du $\mathrm{XX}^{\mathrm{e}}$ siècle, des physiciens comme Bohr et surtout Heisenberg ${ }^{53}$ insistaient sur le caractère essentiellement déterminant du mode d'observation propre à l'observateur: cela est vrai, tout dépend ensuite des conclusions qu'on en tire, comme on peut le voir avec Bachelard. Aujourd'hui, d'un point de vue radical que je jugerai extra-scientifique, la position de certains sociologues de la science fait de l'activité scientifique une activité purement sociale: j'abandonne ce débat aux spécialistes de la sociologie des sciences qui, d'ailleurs, ne méconnaissent pas tous l'importance ni la valeur du discours épistémologique.

Pour ma part, je relève essentiellement qu'au camp des anti-réalistes contemporains se joignent essentiellement tous ceux qui prétendent trancher entre «observables» et «inobservables», pour accorder à la réalité des dits «observables» une certitude qu'ils n'accordent pas aux prétendus «inobservables ».

53 Cf La nature dans la physique contemporaine, p. 126: «Lorsque nous observons des objets de notre vie quotidienne, le processus physique qui rend possible cette observation ne joue qu'un rôle secondaire. Mais chaque processus d'observation provoque des perturbations considérables dans les particules élémentaires de la matière.» 
Il est vrai qu'une tendance globale se dessine qui voudrait classer le réalisme dans le compartiment des vieilles lunes. La question du réalisme me semble essentielle car elle touche à la réalité tout entière y compris la réalité humaine - ce que les philosophes de l'existence, K. Jaspers ${ }^{54}$ (1883-1969) et M. Heidegger (1885-1976), dénommaient Dasein -, même si cette réalité ne nous est pas perceptible immédiatement ni pleinement dans sa totalité.

D'entrée de jeu, d'une manière générale et d'un point de vue encore tout extérieur, je pense qu'il est difficile sinon impossible de créer de toutes pièces et simultanément autant nos modes d'approche de l'objet que la réalité même de l'objet de cette approche. L'artiste est certainement doué pour accomplir cette double création et il ne s'en prive pas; on peut certes, un instant, supposer que le scientifique en arrive un jour à ce mode de comportement jusqu'à présent propre à l'artiste; cela supposerait que nous vivions dans un milieu différent de la Terre, un monde pour nous sans qualité préalable aucune, dans lequel le scientifique aurait eu le champ libre de créer artificiellement une condition humaine dont la survie serait garantie sous un mode différent et dans un milieu différent qui furent les siens au départ. Si cette idée ne relève pas absolument de l'impossible, elle demeure encore, du moins dans son intégralité, de l'ordre de l'utopie. Même dans cette perspective extra-terrestre et surtout dans la mesure où nous continuons à vivre sur Terre et dans cet univers, la mission du scientifique est précisément de connaître toutes les conditions qui nous sont naturellement données et qui nous sont indispensables pour vivre ou, au contraire, que nous devons combattre pour survivre: par exemple, la réalité des virus est, de cette manière, à prendre au sérieux, tout comme la réalité des radiations cosmiques. L'objet d'étude du scientifique est le monde, les humains compris, même s'il ambitionne de créer un monde artificiel.

Par ailleurs, chaque fois qu'il le souhaite et qu'il lui plaît de réfléchir de cette manière - surtout lorsqu'il compare le discours scientifique aux mythes et aux rites qu'ils perpétuent! - absolument rien ne peut empêcher l'anti-réaliste ou le relativiste de penser que la réalité est une création de la science, c'est-à-dire de l'être humain! Mais il faut dire que cette réflexion ne correspond nullement à notre condition humaine qui ne peut se maintenir et perdurer qu'en obéissant à certaines lois de la nature, inconnues du bon sauvage d'autrefois. Dans tous les cas, la négation de la réalité des objets scientifiques relève d'une attitude qui est ou qui se veut non informée; du moins, qui ne veut pas même entendre raison en ce qui concerne les résultats tangibles qui suivent les applications des énoncés scientifiques dans le monde pratique ${ }^{55}$.

54 Cf K. Jaspers, Psychologie der Weltanschauungen, Heidelberg, Muenchen, Springer Verlag, 1919. Continuant la pensée de Dilthey sur les conceptions du monde, Jaspers anticipa sur les philosophies de l'existence qui se firent connaître en 1927 avec le livre de G. Marcel, Journal métaphysique, Paris, Gallimard; et celui de M. Heidegger, Sein und Zeit, traduit en françait par F. Vezin, Etre et temps, Paris, Gallimard, 1986. Voir A. Kremer-Marietti, Jaspers et la scission de l'être, Paris, Seghers, 1967, 1974; L'Harmattan, 2002; du même auteur, Dilthey et l'anthropologie historique, Paris, Seghers, 1971.

55 Je note que D. Pestre, qui doute de la réalité des objets scientifiques quand il affirme, par ailleurs, que la Nature ne parle jamais («ce sont toujours les hommes qui parlent en son nom»), écrit pourtant dans son Introduction au numéro qu'il a organisé, La science et la guerre in La Recherche, 
D'une part, un raisonnement scientifique à l'œuvre au-delà de la réalité triviale pourrait, pour ceux qui l'ignoreraient ou feindraient de l'ignorer, sembler créer la réalité de ses propres objets; d'autre part, un raisonnement scientifique plus raffiné ou plus évolué, et chaque fois plus indirect, pourrait, tout en s'adossant à la réalité, la présenter sous des aspects divers liés à la diversité de ses méthodes d'approche donnant des niveaux différents de cette même réalité pourtant une: de là à douter de l'existence de cette dernière, le pas serait vite franchi par les relativistes pour qui l'être humain crée de toutes pièces le monde dans lequel il vit en se créant aussi lui-même, en chair et en os ou en circuits dont résulterait un homme bionique. Toutefois, il faut remarquer que pour arriver à cette création totalement artificielle de l'homme et du monde, il faudrait déjà, au départ, connaître le monde et l'homme tels qu'ils furent naturellement structurés, au point de départ. On ne peut modifier sainement que ce que l'on connaît parfaitement; certes on peut modifier monstrueusement ce que l'on ne connaît pas précisément.

Vu du plus grand angle, la question du réalisme présente plusieurs niveaux et je partirai du niveau élémentaire de la perception ordinaire, ainsi que des énoncés perceptuels qui en sont issus : ce qui m'y contraint, c'est l'impact de la discussion entre les réalistes et les anti-réalistes, quand ces derniers admettraient de classer les phénomènes entre «observables» et «inobservables»: les premiers étant «réels» parce qu'ils seraient «observables» par nos sens, tandis que les «inobservables» tomberaient dans le domaine de la non-réalité. Ce débat s'est donc ouvert au XIX ${ }^{\mathrm{e}}$ siècle, chez les physiciens, parmi lesquels Mach $^{56}$ affirmait que la force était un «inobservable», et dès lors un objet sans réalité. C'est alors que F. Brentano ${ }^{57}$ (1838-1917) proposait une théorie de «l'évidence immédiate» et authentique concernant les grands principes logiques et mathématiques, mais encore les dits «énoncés auto-psychologiques » qui décrivent la conscience intérieure. Curieusement, les positions de Mach et de Brentano ont en commun d'accorder leur confiance à la perception ordinaire et d'accréditer l'observation naturelle d'un privilège de certitude dont serait privée «l'observation artificielle», indirecte, concernant les prétendus «inobservables». En effet, dans le premier chapitre de L'analyse des sensations, un livre constamment révisé de 1882 à 1906, Mach appuie l'observation scientifique sur un phénomène d'adaptation:

\begin{abstract}
La science a toujours son origine dans l'adaptation de la pensée à un champ d'expérience défini. Les résultats de l'adaptation sont les éléments de pensée, qui sont capables de représenter le champ tout entier. L'aboutissement est naturellement différent suivant le caractère et l'étendue du champ. Si le champ d'expérience est élargi, ou si plusieurs champs jusqu'à présent séparés sont unis, les éléments familiers, traditionnels ne suffi-
\end{abstract}

hors série, $\mathrm{n}^{\circ}$ 7, avril-juin 2002, p. 6: «A la lecture de ce numéro [...] pour qui regarde les choses telles qu'elles furent historiquement, les sciences apparaissent plutôt comme des systèmes de savoir et de pratiques visant à maîtriser le monde naturel et humain, visant certes à comprendre la nature, mais aussi, et indissociablement, à agir sur elle, à la modifier - et à faire la guerre ». Donc il faut croire que ce dont traite la science est bien la réalité! F. Bacon disait déjà, en rapprochant savoir et pouvoir «Scientia et potentia humana in idem coincidunt».

56 Cf E. Mach, La Mécanique, traduit par Bertrand, Paris, Hermann, 1904 (titre original Die Mechanik in ihrer Entwicklung historisch-kritisch dargestellt, 1883).

57 Cf F. Brentano, Psychologie du point de vue empirique (1874-1911), traduit par M. de Gandillac, Paris, Aubier, 1944. 
sent plus pour le champ étendu. Dans la lutte de l'habitude acquise avec l'effort d'adaptation, des problèmes se posent, qui disparaissent, quand l'adaptation est perfectionnée, pour laisser place nette à d'autres problèmes qui se sont posés entre-temps ${ }^{58}$.

\section{LE FAUX DUALISME OBSERVABLE/INOBSERVABLE}

Aussi suis-je frappée par l'argument que présentent en eux-mêmes les termes «observable» et «inobservable» tels qu'ils sont utilisés par les anti-réalistes acceptant la réalité des «observables» et doutant de la réalité des «inobservables ».

Je remarque que le terme «inobservable»n'est pas un terme scientifique et qu'il ne comporte aucune définition hors du domaine de l'expérience ordinaire dans lequel il ne signifie rien d'autre que «non visible», tandis que le terme «observable» peut être scientifiquement défini, encore qu'il puisse être utilisé aussi à ce que j'appellerai un degré zéro de l'observation, quand il concerne le résultat d'une observation sensible de la perception ordinaire. Aussi, j'ai pu voir que l'obstacle du réalisme n'en était plus un, par exemple quand les instrumentalistes avaient à faire avec des objets observables, dont ils acceptaient sans discussion la réalité.

J'ai suivi, en effet, avec un grand intérêt la démonstration développée par Pierre Jacob ${ }^{59}$ dans son débat avec les anti-réalistes. Après avoir éliminé comme «auto-réfutantes» les versions «éliminativiste» et «relationnelle» de la vérité, Pierre Jacob s'attarde à la version relativiste, dite «thèse de la pluralité des mondes » ou «thèse de l'incommensurabilité ${ }^{60}$ avancée par Kuhn ${ }^{61}$ et P. Feyerabend $^{62}$ (1924-1994). Au lieu de postuler la «pluralité des mondes», mieux vaut, selon Pierre Jacob, admettre la sous-détermination des théories par les données expérimentales: c'est le résultat d'une fatale incertitude inductive. Il a raison de dire que ce qu'il faut pouvoir comprendre, ce sont moins les désaccords entre les scientifiques que leurs convergences. D'une part, une théorie scientifique possède une «condition de vérité» et une valeur de vérité: elle est vraie ou fausse; et, d'autre part, les raisons d'adopter une théorie scientifique sont ipso facto des raisons de la croire vraie.

Ainsi, Pierre Jacob remarque, à l'encontre de l'instrumentalisme épistémique, que ses partisans distinguent curieusement entre «le fait de croire vraie une théorie et le fait de la croire empiriquement adéquate» parce que, selon ce point de

58 Ma traduction de: E. Mach, The Analysis of Sensations (1897), translation by C. M. Williams \& S. Waterlow, Dover Edition, 1959, First Chapter, Section 14.

59 Working paper 99-6, voir le site: http://www.isc.cnrs.fr, cf. «Le Débat», 102, 1998.

60 Je renvoie à l'excellent livre de J.-C. Sacchi, Sur le développement des théories scientifiques. De l'aporie de l' incommensurabilité à la dimension pragmatique de la découverte, Paris, L'Harmattan, 1999. J'ai moi-même abordé la question dans le chapitre Les sciences dans le miroir de l' histoire de mon livre, Philosophie des sciences de la nature.

61 Cf La structure des révolutions scientifiques.

62 Cf P. Feyerabend, Contre la méthode. Esquisse d'une théorie anarchiste de la connaissance (1975), traduit par B. Jurdant \& A. Schlumberger, Paris, Seuil, 1979. 
vue, les théories ne servent qu'à permettre de dériver des prédictions empiriques et à expliquer les phénomènes observables: même si elles y réussissent, ils ne croient pas nécessaire de les tenir pour vraies. Or, selon Pierre Jacob, adopter une théorie pour empiriquement adéquate et croire vraies toutes ses conséquences observables, c'est considérer la théorie globalement: et, dans ce cas, ne pas la considérer comme vraie ce serait se priver des justifications pour lesquelles elle est valablement utilisée. Notons déjà le préjugé favorable de ce qui est ici dit «observable » et donc réel en même temps que vrai, par rapport à la théorie cependant non reconnue vraie.

Voyons maintenant - également analysé par Pierre Jacob-1'instrumentalisme sémantique qui suspecte plus directement les entités ou processus «non directement observables à l'œil nu »: d'où la position de R. Carnap (1891-1970), en tant qu'empiriste logique que l'on peut considérer comme représentatif de l'instrumentalisme sémantique actuel, dans la mesure où il suspectait l'existence de l'électron, non directement observable, le terme «électron» restant sans référence. La proposition incluant le terme «électron» était aux yeux de Carnap une règle d'inférence qui permettait la prédiction sur le comportement de l'aiguille d'un galvanomètre, tout en demeurant ni vraie ni fausse. Seules les prédictions observationnelles obéissaient pour lui à une condition de vérité, mais non les lois théoriques qui en étaient a priori dépourvues tout en étant admises pour être cependant conformes à la réalité.

D'ailleurs, toutes ces positions instrumentalistes partent d'un point de vue particulier concernant le concept même de vérité, les théories n'étant vues que comme des instruments de vérité; ce qui est une position diamétralement opposée à celle de Bachelard pour qui, tout au contraire, un instrument proprement dit est une théorie vraie qui a été matérialisée. Ce qui revient à dire, en ce qui concerne les instrumentalistes, que la théorie incorporée dans l'instrument auquel ils acceptent de se fier doit nécessairement avoir à leurs yeux une quelconque valeur de vérité, sans laquelle ils ne pourraient se fier aux résultats donnés par l'instrument, résultats qu'ils disent reconnaître comme observables et donc vrais.

\section{CONTRE LE RÉALISME IMMÉDIAT DE LA PERCEPTION ORDINAIRE}

C'est pourquoi, je me permettrai de partir de la définition du sens commun que donne le Petit Larousse, une définition extérieure à la science, et qui s'énonce justement comme un énoncé auto-psychologique sur la base d'un fait de conscience, puisque le réalisme y est dit brièvement mais nettement une: «disposition à voir la réalité telle qu' elle est et à agir en conséquence».

Voyons les termes employés. Pour la «disposition» aperçue, on peut être banalement d'accord, on peut et l'on doit même toujours supposer qu'elle existe, on souhaiterait même qu'elle existe! Ne croit-on pas déjà qu'elle existe, chez soimême comme chez autrui, quand on cherche à communiquer sincèrement avec des interlocuteurs sincères? Du désir nous passons donc aisément à la croyance, mais nous découvrons que ce qui était désir et croyance ne l'étaient que parce qu'une communication - qu'elle soit un monologue ou un dialogue - nécessite le présupposé intellectuel que la réalité du message soit prise en considération, dans 
sa forme et dans son contenu, par celui auquel il est destiné. D'une part, si donc elle est supposée exister, connaît-on chez les uns et les autres la qualité de cette disposition: ce qu'elle est? D'autre part, connaît-on le degré de clairvoyance de cette «disposition à voir»: son intensité? Surtout, connaît-on, ou peut-on réellement connaître la nature de la «réalité» visée: elle serait vue, mais surtout «vue telle qu' elle est»; sait-on sous quel angle, selon quelle méthode, nous pouvons ou d'autres que nous peuvent la voir «telle qu' elle est»? Outre un présupposé de la «disposition à voir», nous avons maintenant un autre présupposé, celui de la vision permettant de voir la «réalité», et même «la réalité telle qu' elle est». Mais la vision de la «réalité telle qu' elle est», qui est une vision particulière, n'a-t-elle pas elle-même ses présupposés? Outre le présupposé de la disposition à voir, puis le présupposé de la vision quelconque d'un objet quelconque, nous avons maintenant le ou les présupposés de la vision de la réalité telle qu' elle est, et qui n'est pas n'importe quelle vision, puisque c'est celle de la «réalité telle qu'elle est» et non «telle qu'elle n'est pas».

Supposons enfin que soit vue, authentiquement et sans erreur avec toutes les précautions nécessaires, «la réalité telle qu'elle est», alors saurons-nous y faire face? Comment «agir en conséquence» de la «réalité telle qu' elle est»? Un autre savoir s'impose ici, outre le savoir de la réalité, et qui est le savoir de l'action qui dépend immédiatement du savoir de la réalité: saurons-nous accomplir ce genre d'action qui consiste «à agir en conséquence»? Vous feriez d'une façon, et moi peut-être d'une autre: où est la bonne façon d' «agir en conséquence» devant la «réalité telle qu'elle est»? Mais il y a de fortes chances pour que l'action et le savoir qu'elle exige dérivent avant tout de la réalité et du savoir qu'elle implique. Nous serions privés d'agir avec la conséquence appropriée si nous ignorions ce qu'est «la réalité telle qu' elle est». En vue de cette action conséquente et appropriée, notre premier devoir consiste en la connaissance de la «réalité telle qu'elle est», pour laquelle nous avons présupposé une disposition première qui est en fait une disposition à «voir la réalité», mais, en outre, à la voir «telle qu' elle est».

Que d'exigences, pour aboutir à la constatation indiscutable que, sans connaissance de la «réalité telle qu' elle est», je ne puis agir en conséquence ! Et toutes ces difficultés peuvent concerner le «réalisme naïf » qui impliquerait une disposition humaine avec toutes ses conséquences, la plus déterminante de toutes étant la connaissance de la «réalité telle qu'elle est». Quand les instrumentalistes d'aujourd'hui ou les positivistes d'autrefois, ou encore leurs contemporains les phénoménistes, constatent comme «observables » ou «inobservables » certains phénomènes, ils ne le font que sur la base d'un tel «réalisme naïf» auquel ils n'échappent pas malgré tous leurs arguments sophistiqués. La conclusion est qu'on ne peut observer impunément.

Dès lors, on peut donc supposer un instant que le «réalisme scientifique » doit être beaucoup plus complexe mais qu'il comporte globalement les mêmes tenants et aboutissants: c'est-à-dire la prise de conscience, non pas vulgaire mais scientifique, d'une réalité quelle qu'elle soit, mais d' une réalité « telle qu'elle est scientifiquement», afin de pouvoir toujours et encore «agir en conséquence», cette conséquence scientifique étant avant tout la conséquence de la connaissance scientifique de la «réalité telle qu'elle est scientifiquement».

Le niveau de l'expérience ordinaire aboutit à des énoncés perceptuels, qui ne dérivent ici que d'une définition d'un dictionnaire universel destiné à tous les 
usages, sans que soit présupposée aucune spécialité ni aucune compétence particulière des usagers. "Philosophiquement», la définition du Larousse nous apprend que le « réalisme » n'est pas chose facile, même si nous nous en tenons au niveau de la perception ordinaire. D'ailleurs, dès qu'il s'agit d'établir un constat d'utilité publique, concernant par exemple un accident de la route, nous savons que certaines règles d'usage sont imposées par les institutions de police et par les compagnies d'assurances, afin qu'il soit procédé à un enregistrement fiable des déclarations et des faits invoqués. Rien ne dit toutefois que certaines déclarations ou constatations ne soient falsifiées dès le départ: on a souvent remarqué, au cours d'affaires criminelles, que de nombreuses enquêtes policières ont été, dès les premiers interrogatoires, mal dirigées ou menées à partir de bases erronées, au point que des questions qui venaient à se poser ultérieurement ne puissent plus être élucidées, justement du fait de l'absence des données qui avaient échappé aux observateurs de la première heure, faute d'y avoir pensé. Mais il ne suffit pas d'y penser, encore faut-il avoir mis en œuvre un équipement soit intellectuel soit matériel, ce dernier découlant d'une précédente approche orientée dans la même direction, car l'instrument ou l'appareil n'est autre que de l'expérience et du savoir matérialisés: il perçoit à notre place la réalité parce qu'il a été lui-même pensé pour la percevoir comme il le fait.

Or, les questions auxquelles nous soumettons la définition du «réalisme » par le Petit Larousse sont loin d'être vulgaires. En les posant, c'est à la critique popperienne du psychologisme, aux suggestions de Brentano sur le comment de la perception ordinaire, mais c'est surtout à Spinoza que je suis reconduite - Spinoza aux distinctions duquel je dois me consacrer pour justifier un mode de perception. Voulant atteindre la réalité, qui était donc aussi son problème, il fait une analyse très simple des modes de perception de cette même réalité: je renvoie au début de son Traité de la réforme de l'entendement, qui, pour dater de 1661, n'en présente pas moins matière à une réflexion très moderne.

Par conséquent, pour Spinoza, l'examen de la perception devait permettre une réforme de l'entendement dans la perspective de la connaissance authentique de la réalité. A cet effet, Spinoza distingue donc divers modes de perception de la réalité: quatre qui sont déterminés dans une progression allant du mode du «ouïdire » au mode de l'«essence», c'est-à-dire d'un mode confus vers un mode clair. En l'occurrence, la référence sous-jacente à un verre que l'artisan polirait et dégrossirait progressivement n'est pas interdite si l'on se souvient du métier de l'optique qui était celui du philosophe. Et tels sont les modes mis en évidence par Spinoza que je cite ${ }^{63}$ :

D'abord, le premier mode: « une perception acquise par ouï-dire par le moyen d'un signe conventionnel»: c'est le niveau de la perception ordinaire ou vulgaire, par exemple liée à la coutume ou à la narration, en tout cas non scientifique.

\footnotetext{
Ensuite, le second mode: «une perception acquise par expérience vague » - c'est-à-dire non déterminée par l'entendement, et probablement non contredite par aucune autre, donc demeurée inébranlée: c'est le niveau de la perception ordinaire ou vulgaire provenant de l'habitude et de la pratique, et nullement scientifique.

Ou bien, le troisième mode: «une perception où l'essence d'une chose se conclut d'une chose, mais non adéquatement»: c'est l'expérience d'un cogito naturel, inexpérimenté
}

63 B. Spinoza, Euvres, t. I, traduit et annoté par C. Appuhn, Paris, Garnier, 1964, p. 231. 


\begin{abstract}
dans les modes d'observer et de raisonner: inculte en ce qui concerne la question à laquelle il prétend répondre, et donc non scientifique.

Enfin, je cite le quatrième mode: «une perception dans laquelle une chose est connue par sa seule essence ou par la reconnaissance de sa cause prochaine»: notons que «sa seule essence» concernerait la définition liée à un savoir [qui pouvait être, pour Spinoza, encore métaphysique]; mais la «reconnaissance par la cause prochaine» implique une connaissance liée à l'observation scientifique et au raisonnement qu'elle a impliqué, de telle sorte qu'a été conclu ou déduit un rapport de causalité.
\end{abstract}

Ensuite, le philosophe donne des exemples illustrant ses distinctions. Reprenons, à travers ces exemples, les quatre points énoncés:

1) c'est par ouï-dire qu'il connaît sa date de naissance;

2) par expérience vague, il sait que l'eau éteint le feu, également toutes les choses apprises par l'expérience de la vie;

3) ensuite, dans la conclusion d'une chose à une autre, il note que l'imagination peut produire des confusions: c'est la répétition de la même sensation du corps qui lui fait conclure à l'union de l'âme et du corps, union considérée comme la cause de cette sensation;

4) enfin, la perception d'une chose par sa seule essence nous permet de conclure quelque autre chose: un exemple de Spinoza est que la connaissance de l'essence de l'âme permet de conclure qu'elle est unie au corps; mais c'est aussi de cette façon que nous connaissons que deux et trois font cinq.

Si l'épistémologie peut se donner le double objet d'analyser les étapes de la pensée scientifique et d'expliquer, en outre, les mécanismes intellectuels propres à l'accomplissement de cette pensée, la réalité visée répondra aux méthodes d'approche mises en jeu. Nous pouvons retenir des distinctions de Spinoza la possibilité d'une marche progressive vers la réalité, sans faire une enquête sur l'enquête ni encore une enquête sur l'enquête de l'enquête: ce dont il se défendait, car les unes et les autres seraient sans fin ${ }^{64}$.

On peut, certes, discuter la distinction des quatre modes et leurs exemples. On peut évidemment retrouver le «signe conventionnel» dans tous les modes de perception, quand on ne vérifie ni un pseudo-savoir ni une pseudo-science. De plus, les deux derniers modes posent des problèmes propres aux jugements en tant que tels: l'un, le problème des jugements empiriques à propos duquel Hume nous a fait savoir qu'aucune connaissance certaine n'était à tirer de l'expérience; et l'autre, le problème des jugements synthétiques a priori, que Kant pose et résout en pensant rendre ainsi la connaissance scientifique possible. On a longtemps cru voir que Hume condamnait les sciences de la nature à se maintenir sur le troisième mode de perception en lui refusant le quatrième mode qu'il réservait aux mathématiques: en fait, sur la base d'une observation qui serait susceptible de nous tromper, il permettait néanmoins l'usage du calcul des probabilités. En ce qui concerne Kant, qui tentait de prouver que la connaissance de la nature relève du quatrième mode, l'usage des mathématiques dans la physique lui donne totale-

64 Op. cit., p. 236. 
ment raison si l'on admet que le développement des mathématiques est à la fois synthétique (nullement analytique, comme le veulent les logiciens) - puisqu'il progresse par accroissement ou par intégration d'éléments toujours nouveaux - et a priori, c'est-à-dire s'organisant selon la création de calculs divers, suscités en vue de se conformer à un objet déterminé mais se déployant du fait de leur logique propre.

Outre cette exposition classique des modes de la perception, nous ne pouvons ignorer l'épistémologie génétique ${ }^{65}$ qu'ignorait le Cercle de Vienne et que veulent ignorer nos logiciens contemporains; l'épistémologie génétique nous apprend que le second mode, que Spinoza appelle «perception acquise par expérience vague », préside, en fait au tout commencement de ce qui deviendra l'opération logique pure. Car même la vérité $2+3=5$ a dû requérir un appel à l'expérience avant de donner lieu à un maniement purement déductif. Et, bien que ces coordinations ne soient pas dans les choses - ce sur quoi insiste Hume -, elles y ont été introduites par l'action de la vie quotidienne pour ses besoins propres. Ainsi, l'enfant qui compte dix cailloux, et découvre qu'ils sont toujours dix, alors qu'il en permute l'ordre, expérimente non pas sur les cailloux, mais sur ses propres actions d'ordonner et de dénombrer. En tant que sujet connaissant, il abstrait à partir de ses propres actions, et non à partir de l'objet: c'est d'ailleurs aussi dans ce sens que Kant dit, en suivant la leçon de Hume, que la connaissance vient avec l'expérience, non pas de l'expérience. En effet, à première vue, l'objet ne semble comporter lui-même ni ordre ni nombre; et toutes ces coordinations de mouvements ne seront plus utiles quand l'enfant de huit ans les aura intériorisées et qu'il déduira par simple opération logique. De plus, le signe conventionnel intervient dès que l'enfant connaît la nomenclature numérique cardinale et ordinale. N'estce pas par ouï-dire que l'enfant apprend la suite des nombres cardinaux? D'où l'utilisation du premier mode de perception dans l'apprentissage de ce qui deviendra le quatrième mode.

Entre le troisième et le quatrième mode, il peut y avoir la différence qu'il y a entre un contenu de l'expérience et la nécessité de ce contenu; c'est ce qui arrêta Hume devant le problème de la nécessité des vérités des sciences de la nature; et c'est ce que Kant se donne pour tâche de justifier dans le cadre des catégories servant à la connaissance des choses en tant que ces choses sont considérées comme des objets d'expérience possible. C'est pourquoi Kant part du fait symbolique que toute opération de l'entendement repose sur une fonction, c'est-à-dire l'unité de l'acte qui consiste à réunir diverses représentations sous une représentation commune, le jugement, qui est «la faculté de penser le particulier comme contenu dans le général » ${ }^{66}$ : le général étant donné, le jugement y subsume le particulier et est déterminant; le particulier seul étant donné, le jugement n'est que réfléchissant. Or, connaître scientifiquement, c'est déterminer; sinon le jugement ne fait que « réfléchir». Les lois de l'esprit convergent toujours vers la constitution d'une unité qui est un «système articulé » ${ }^{67}$.

65 Voir J. Piaget, Introduction à l'épistémologie génétique, Paris, PUF, 1974 (1950).

66 Cf I. Kant, Critique du jugement, traduit par Gibelin, Paris, Vrin, 1960, Introduction, IV, p. 20 (titre original Kritik der Urteilskraft, 1790).

67 Cf I. Kant, Critique de la raison pure (1781, 1787), Méthodologie transcendantale, Chapitre III: L'architectonique de la raison pure; Kantische Originalausgabe von 1787, p. 861. La connais- 
Si j'insiste sur les difficultés de l'observation pourtant souvent conduite selon des règles rationnelles, c'est pour atténuer la prétention au caractère absolu de certaines données concernant les dits «observables», sur lesquelles on s'appuie aujourd'hui encore, sans préciser de quelle façon ils sont observables et pour refuser, par contraste, toute certitude de réalité à de prétendus «inobservables », malgré les mesures faites sur ces mêmes dits «inobservables»: en effet, l'argument majeur des antiréalistes ou plus simplement des «phénoménalistes » du siècle dernier comme Mach, concerne essentiellement la difficulté qu'il y a à asseoir une certitude de réalité liée à de prétendus «inobservables», qui ne le sont en fait que dans la perception ordinaire, et en justifiant cette position par la référence à la prétendue certitude absolue née du traitement des dits «observables». Je refuse cette argumentation et, tout au contraire, je mets en doute la fiabilité toute relative, et trop facilement accordée aux «observables » du premier degré (ou du degré zéro) parce que je ne trouve pas que l'argumentation de la «perception ordinaire» puisse figurer comme un bon argument à valoir contre la réalité des supposés «inobservables». Nos sens peuvent nous tromper tout autant que nos instruments de mesure. Ce qui veut dire que la mesure des «observables» doit être mise à égalité avec la mesure des prétendus «inobservables» pour autant que les uns et les autres soient mesurés - or, les supposés «inobservables» de la perception ordinaire sont effectivement mesurés en physique quantique, où on les appelle précisément des «observables» puisque: «La mécanique quantique ne s'intéresse qu'aux 'observables', qui sont des grandeurs physiques, résultats possibles des observations effectuées sur un objet quantique.» ${ }^{68}$ Mieux encore: «Les propriétés d'un système physique ne sont observables que lors de l'interaction de ce système avec un dispositif qui joue le rôle d'un instrument de mesure.» ${ }^{69}$

Les «observables» de la physique quantique ont en commun avec les observables de la mécanique classique d'être mesurés: ce qui les réunit n'est autre que le concept du quantitatif, nécessaire à l'édification de la science physique.

C'est pourquoi je reviens sur l'examen des positions phénoménalistes qui affirmaient que seuls les énoncés portant sur des phénomènes observables devaient être considérés comme une connaissance scientifique valide. Et ils en tiraient pour conséquence de donner un but particulier à la science: qui était d'identifier, de classifier et de lier les phénomènes observables entre eux. Du point de vue des phénoménalistes, se limiter aux seuls faits observables conduisait à une certitude permanente et même absolue. Un fait expérimental identifié devenait ainsi une connaissance immuable, car tout expérimentateur répétant ladite mesure ne pouvait qu'obtenir le même résultat. Au contraire, les théories abstraites faisant appel à des entités non-observables par les sens, identiquement mesurées, et qui

sance ne s'arrête pas au niveau de l'Esthétique transcendantale et des intuitions de l'espace et du temps: l'Analytique transcendantale opère en tant que les fonctions de l'esprit sont déjà à l'œuvre; même si l'objet est donné sous sa forme sensible dans l'intuition, il faut toujours lui ajouter le concept de l'objet, situé au-delà de la perception ordinaire. Voir les deux chapitres, VI L'abîme de la raison et VII Contingence et nécessité, dans A. Kremer-Marietti, La raison créatrice. Moderne ou postmoderne, Paris, Kimé, 1996.

68 Cf G. Lochak, S. Diner \& D. Fargue, L'objet quantique. Comment l' esprit vient aux atomes, Paris, Flammarion, Collection Champs Flammarion, 1989, p. 216.

69

Ibid. 
seraient échafaudées à partir de ces mesures, ne seraient pas totalement fiables à leurs yeux. Ernst Mach, qui a représenté un tel mouvement anti-réaliste, a pourtant contribué à faire avancer la mécanique, l'hydrodynamique, l'électricité, la thermodynamique, et même l'esthétique. Il attribuait aux lois de la nature l'objet de synthétiser l'ensemble de ce qui était perçu et expérimenté; pour lui, une bonne théorie devait être concise, économe en termes de concepts, et simple. Dans son ouvrage sur le développement de la mécanique ${ }^{70}$, Mach démontrait que la masse et la force sont des concepts qui n'entrent jamais simultanément de manière descriptive dans les expériences, mais seulement à travers l'accélération; ainsi, la force, faisant partie des entités supposées non-observables, n'aurait aucune réalité objective, et n'aurait qu'une vertu simplificatrice dans l'énoncé des lois physiques. Pour Mach, les phénomènes ultimes étant les sensations, les phénomènes physiques n'étaient qu'un ensemble d'éléments sensoriels apparaissant sous la forme de sensations: "les 'sensations', seules choses accessibles à la connaissance. Les couleurs, les sons, les espaces, les temps... sont pour nous, au demeurant, les éléments ultimes...» ${ }^{71}$ Considérés les uns par rapport aux autres, et selon un observateur, les phénomènes apparaissaient comme des propriétés ou des qualités des corps: les connaissances concernant un corps en tant que tel n'étaient pas accessibles: seules, les connaissances sur les éléments sensoriels qui le composent l'étaient; et cela, du seul fait que ces connaissances nous parviennent par nos sensations qui sont le seul moyen que nous ayons de les appréhender.

Si j'évoque cette position d'un physicien par ailleurs remarquable, c'est parce qu'actuellement le même argument relatif à la différence des «inobservables » et des «observables » a, me semble-t-il, toujours cours. L'argument me paraît mettre en cause le recours à la perception, une perception, comprise comme la garantie de la réalité de l'objet scientifique. Dans ce cas, on tombe au niveau trivial de la conscience vulgaire, qui dénomme «réalité» le monde auquel les sens ont accès.

Certes, c'est avec les sens, et surtout le sens de la vue, que les chercheurs relèvent les mesures sur les divers appareils d'expérience utilisés dans le laboratoire. Mais, dans ce cas, nos sens n'ont qu'un rôle limité: le gros du travail est accompli par ces appareils, que l'on peut considérer comme du savoir scientifique matérialisé. L'œil qui relève les résultats des mesures de ces appareils a un rôle passif, même si enregistrer et comprendre les résultats exigent davantage qu'enregistrer, c'est-à-dire aussi la connaissance du savoir impliqué dans la structure de l'appareil: quelles lois ces appareils représentent matériellement et ainsi quelles lois opèrent dans la quête d'information à laquelle procède l'appareil.

Dans la mesure où «observables » et «inobservables» sont mis en concours et sur le pied d'égalité lié au concept de quantité, je dirai qu'ils le sont en référence aux appareils qui les examinent avec la médiation d'un observateur qui n'observe que les résultats des mesures de ces appareils en relation avec ce qu'il considère comme étant des «observables». Dans un second temps, l'observateur donne une interprétation scientifique adéquate, sur la base des mesures pratiquées par un appareil qui est lui-même le résultat d'un savoir scientifique.

E. Mach, Die Mechanik in ihrer Entwicklung historich-kritisch dargestellt, 1883.

71 E. Mach, L'analyse des sensations, traduit par F. Eggers \& J.-M. Monnoyer, Nîmes, J. Chambon, 1996 (1882), p. 31. 
Comme E. Cassirer (1874-1945), je dirai qu'ici ce ne sont pas nos sensations qui sont en cause, mais, avec l'aide de tous les instruments du laboratoire, il s'agit essentiellement de «médiations symboliques»: et cela concerne également les dits «observables » qui bénéficient du privilège de notre perception.

Croire dans le privilège de la perception immédiate, c'est se mettre au niveau d'un réalisme immédiat ou naïf, qui est celui de la perception ordinaire. Car un corps ne se définit pas scientifiquement par son apparence sensible; les données de l'expérience sensible ne sont pas les data de la physique. Un corps se définit par la médiation des concepts, c'est-à-dire par son poids atomique, sa chaleur spécifique, son indice de réfraction, son indice d'absorption, sa conductibilité électrique, sa susceptibilité magnétique ${ }^{72}$, etc.

Ce qui veut dire qu'il ne faut pas confondre la «réalité» avec la réalité tout court sur le pied d'un «réalisme immédiat», selon l'expression que lui donne Bachelard que je cite:

\begin{abstract}
Tôt ou tard, c'est la pensée scientifique qui deviendra le thème fondamental de la polémique philosophique; cette pensée conduira à substituer aux métaphysiques intuitives et immédiates les métaphysiques discursives objectivement rectifiées. A suivre ces rectifications, on se convainc par exemple qu'un réalisme qui a rencontré le doute scientifique ne peut plus être de même espèce que le réalisme immédiat. On se convainc également qu'un rationalisme qui a corrigé des jugements a priori, comme ce fut le cas dans les nouvelles extensions de la géométrie, ne peut plus être un rationalisme fermé. Il y aurait donc intérêt, croyons-nous, à prendre la philosophie scientifique en elle-même, à en juger sans idées préconçues, en dehors même des obligations trop strictes du vocabulaire philosophique traditionnel. La science crée en effet de la philosophie. Le philosophe doit donc infléchir son langage pour traduire la pensée contemporaine dans sa souplesse et sa mobilité. Il doit aussi respecter cette étrange ambiguïté qui veut que toute pensée scientifique s'interprète à la fois dans le langage réaliste et dans le langage rationaliste. Peutêtre alors devrait-on prendre comme une première leçon à méditer, comme un fait à expliquer, cette impureté métaphysique entraînée par le double sens de la preuve scientifique qui s'affirme dans l'expérience aussi bien que dans le raisonnement, à la fois dans un contact avec la réalité et dans une référence à la raison ${ }^{73}$.
\end{abstract}

Autrement dit, aujourd'hui nous pouvons déclarer: «Nous vivons dans un monde quantique et tout le monde l'ignore, en dehors d'une minorité de spécialistes $»^{74}$.

\title{
VI. LA MESURE DES GRANDEURS EN PHYSIQUE QUANTIQUE
}

Dans le travail de recherche, le réalisme scientifique est une attitude nécessaire en tant que «réalisme physique ${ }^{75}$ : «L'épistémologie spontanée des scienti-

72 Cf. E. Cassirer, La philosophie des formes symboliques, t. III, La phénoménologie de la connaissance, traduit par O. Hansen-Love, J. Lacoste \& C. Frontyl, Paris, Minuit, 1972 (1929), p. 482. Voir le chapitre V, Comment Cassirer traite le problème de la symbolisation, dans A. KremerMarietti, La raison créatrice, pp. 71-87.

73 Le nouvel esprit scientifique, p. 3.

74 Lochak, Diner \& Fargue, L'objet quantique, p. 7.

75 Je reprends l'indication donnée par le titre de la conférence de Bricmont et Sokal, Physical Realism from a Physical Perspective, au Colloque de Bielefeld de Juin 2001, Welt und Wissen Monde et Savoir - World and Knowledge. 
fiques - qui anime leur travail, à l'exception de ce qu'ils peuvent dire en philosophant - est donc un réalisme 'à l'emporte-pièce' (rough-and-ready): le but de la science est de découvrir (sous certains aspects) comment les choses sont réellement. Plus précisément, le but de la science est de donner une description vraie (ou approximativement vraie) de la réalité ${ }^{76}$. Dans leur conférence de Bielefeld (2001), les auteurs Sokal et Bricmont précisent ensuite que les théories scientifiques sont vraies ou fausses mais que leur vérité ou fausseté est littérale, nullement métaphorique: elle ne saurait dépendre ni de la manière dont les scientifiques procèdent, ni de la structure de leur esprit, ni davantage de la société dans laquelle ils vivent.

Qu'est-ce que la mécanique quantique? Les manuels nous apprennent que ce n'est pas une description de l'objet quantique en lui-même, «mais un calcul des résultats des observations possibles ${ }^{77}$, reposant sur l'équation de Schrödinger, qui décrit «l'évolution temporelle de l'état d'un objet quantique représenté par une fonction d'onde $»^{78}$. Aussi: «Les lois de la mécanique quantique donnent une description statistique de la réalité microphysique.» ${ }^{79}$ L'instrument de mesure et le calcul y sont donc essentiels; c'est pourquoi la mécanique quantique ne s'intéresse qu'aux «observables »: c'est-à-dire aux grandeur physiques et à leur représentation par un opérateur, le phénomène impliquant son observation. L'interprétation de la mécanique quantique donne lieu à deux prises de position: soit celle de ceux qui pensent que «la réalité physique s'épuise dans la formalisation des observations possibles ${ }^{80}$; soit celle de ceux qui pensent «qu'il existe une réalité en soi, en dehors de toute observation, mais qui échappe à la théorie actuelle ${ }^{81}$. La seconde position est réaliste; la première position peut l'être à condition de préciser s'il y a quelque chose derrière ces formalisations : c'est à quoi l'article de J. Bricmont, «What is the meaning of the wave function? $\gg^{82}$ me semble répondre.

En mécanique quantique, on sait qu'après avoir fait une mesure sur un système, en général on a modifié l'état de ce système; d'une part, si l'on fait une mesure sur un seul système, l'information que l'on obtient est une information sur l'état du système après la mesure; d'autre part, ce n'est que si l'on effectue cette mesure sur $\mathrm{N}$ systèmes préparés indépendamment mais dans le même état, qu'on obtient une information sur cet état avant la mesure. Ce qui veut dire qu'avant la mesure le système est dans un certain état $\Psi(\mathbf{r}, \mathrm{t})$. Mais immédiatement après une mesure de la grandeur physique A ayant donné le résultat aa, on est certain que le système se trouve dans un nouvel état $\varphi_{\mathrm{a}}(\mathbf{r}, \mathrm{t})$, état propre de $\mathrm{A}$ correspondant à la valeur propre de a. L'acte de mesure a instantanément changé la fonction d'onde

\footnotetext{
76 Ma traduction; voir Physical Realism from a Physical Perspective, p. 6 de l'exemplaire dactylographié.

77 Lochak, Diner \& Fargue, L'objet quantique, op. cit., p. 216.

78 Op. cit., p. 222.

79 Op. cit., p. 213.

Op. cit., p. 210.

81 Ibid.

82 J. Bricmont, What is the meaning of the wave function?, first published in J.-M. Frere, M. Henneaux, A. Sevrin \& Ph. Spindel (eds), Fundamental interactions: from symmetries to black holes, Conference held on the occasion of the «Eméritat» of François Englert, Université Libre de Bruxelles, Brussels, 1999, pp. 53-67.
} 
de façon irréversible. On dit qu'il y a réduction du paquet d'ondes ${ }^{83}$. On peut décrire quantiquement l'appareil de mesure, et démontrer la réduction du paquet d'ondes: reste le problème de la prise de conscience du résultat de la mesure par l'observateur ${ }^{84}$.

Le problème étant posé, par exemple, de savoir ce que signifie la fonction d'onde $^{85}$, «concept le plus fondamental de notre théorie physique la plus fondamentale ${ }^{86}$, le chercheur doit dire de quoi sont les probabilités, quand la quantité correspondant à l' «observable» A est mesurée et lorsqu'on peut écrire de la fonction d'onde $\Psi$ :

$$
\Psi=\sum \operatorname{ci} \phi I(1,1)
$$

«où les vecteurs $\phi \mathrm{i}$ sont les vecteurs propres de $\mathrm{A}$, avec des valeurs propres $\lambda \mathrm{i}$ : $\mathrm{A} \phi=\lambda \mathrm{i} \phi \mathrm{i}(1,2)$

Alors, $\Psi$ détermine les coefficients $C$ i et les nombres $[C \mathrm{i}]^{2}$ sont les probabilités pour que la valeur propre $\lambda i$ soit trouvée quand la quantité correspondant à $A$ est 'mesurée'»"

Deux solutions se présentent à J. Bricmont, l'une littérale et l'autre implicite: «La signification littérale dit que les probabilités ne réfèrent qu'aux probabilités de l'expertise accomplies dans le laboratoire ${ }^{88}$; alors rien n'est dit de l'état du monde à l'extérieur du laboratoire et l'on peut dire que «la particule et l'appareil forment un 'tout inséparable'» ${ }^{89}$. Quant à la conception implicite, elle permet de dire: «la 'mesure' signifie réellement une mesure, c'est-à-dire que les expériences révèlent plutôt qu'elles ne créent les propriétés existantes du système ${ }^{90}$. J. Bricmont poursuit: «Suivant cette conception, les particules ont des propriétés telles que la position, le moment, le moment angulaire et, quand nous mesurons un observable $\mathrm{A}$, nous découvrons simplement la valeur que le système a assigné à cet observable indépendamment de toute mesure. ${ }^{91} \mathrm{~J}$. Bricmont fait remarquer que, dans cette interprétation, le statut conceptuel des probabilités est assez semblable à celui qu'elles ont dans la physique classique. La différence s'impose: à savoir que les propriétés restent inconnaissables «parce que mesurer une propriété change la fonction d'onde (suivant le 'postulat du collapse') et dès lors changent les propriétés qui sont associées aux opérateurs qui ne commutent pas avec celles qui sont mesurées $»^{92}$. C'est, nous dit J. Bricmont, la conception implicite qui se tient derrière les formules qui affirment, par exemple, «la mécanique

Cf J.-L. Basdevant, J. Dalibard, Physique quantique, Paris, Ellipses, AUPELF/UREF, 1998, p. 74.

Op. cit., p. 75.

Cf J. Bricmont, What is the meaning of the wave function?

What is the meaning of the wave function?; notre référence va à l'article dactylographié, pp. 1-15, voir p. 1.

Op. cit., p. 2.

Ibid.

Ibid.

What is the meaning of the wave function?, p. 3.

Ibid.

Ibid. 
quantique n'a pas à faire avec la nature, mais avec la connaissance que nous en avons ${ }^{93}$. Or, cette position est, selon J. Bricmont, «logiquement inconsistante». Développant les théorèmes sur l'inexistence des variables cachées, J. Bricmont explique que, selon une sottise historique, une variable qui n'est pas la fonction d'onde elle-même avait été dite une «variable cachée »; or, s'il faut aller au-delà de l'interprétation littérale, il faut statuer qu'il y a quelque chose qui «existe» en plus de la fonction d'onde, elle-même non visible mais inférée comme tous les concepts théoriques. J. S. Bell (né en 1928) appelle des «beable» ces objets: «Ce que les théorèmes sur les variables non-cachées disent, c'est simplement qu'il ne peut y avoir de beable correspondant à tous les observables (ou même à certaines classes d'observables) ${ }^{94}$.

J. Bricmont fait remarquer que c'est la localité qui impliquait des variables cachées, mais ces dernières auxquelles s'ajoute la statistique (lorsque des angles différents sont mesurés) impliquent une contradiction. La source du problème ne vient ni du prétendu réalisme, ni du déterminisme, ni de prétendues variables cachées. Tout vient d'une erreur de départ, à savoir de la première partie de l'argument Einstein, Podolsky et Rosen en $1935^{95}$ («la localité implique des variables cachées»), alors qu'ils soutenaient que le monde est local et concluaient que la mécanique quantique est incomplète. Trente ans après, le théorème de Bell (1964) fut démontré: la conclusion étant que les variables cachées, dont l'existence était impliquée par la formule de la localité proposée par EPR, conduisaient à une contradiction logique. Mais, entre-temps, les physiciens s'étaient rendus aux arguments de Bohr ${ }^{96}$. Le fait est que Bell commença par résumer l'argument EPR, cherchant d'abord à déterminer l'existence de ces variables cachées et donc de la localité, pour aboutir à l'inexistence des variables cachées et à la non-localité; mais ses résultats furent longtemps sous-estimés et négligés, parce qu'ils furent incompris: son premier article ${ }^{97}$ résumant l'argument EPR a été interprété à tort comme partant de variables cachées déterministes.

93 C'est là, d'ailleurs, une remarque que l'on trouve chez Heisenberg, La nature dans la physique contemporaine, p. 126: «les lois naturelles que, dans la théorie des quanta, nous formulons mathématiquement, ne concernent plus les particules élémentaires proprement dites, mais la connaissance que nous en avons.» Op. cit., p. 127: «La conception de la réalité objective des particules élémentaires s'est donc étrangement dissoute, non pas dans le brouillard d'une nouvelle conception de la réalité obscure ou mal comprise, mais dans la clarté transparente d'une mathématique qui ne représente plus le comportement de la particule élémentaire mais la connaissance que nous en possédons ».

94 What is the meaning of the wave function?, p. 7; note de J. Bricmont, What is the meaning of the wave function?, p. 7: «Cela a des conséquences plutôt sérieuses pour les 'histoires décohérentes' touchant aux fondements de la mécanique quantique. Voir Goldstein $(1998,1999)$ pour une discussion plus détaillée».

95 Cf. A. Einstein, B. Podolsky \& N. Rosen, Can quantum mechanical description of reality be considered complete?, in «Phys. Rev.», 1935, $\mathrm{n}^{\circ}$ 47, pp. 777-780.

96 N. Bohr, Can quantum mechanical description of reality be considered complete?, in «Phys. Rev.», 1935, nº 48, pp. 696-702.

97 Article paru dans «Physics», 1935, nº 1, 135, 1965; cité par J. Bricmont, La non-localité et la théorie de Bohm, pp. 1-12, Académie des sciences morales et politiques, online: http://www.asmp.fr; voir p. 3. 
La théorie «purement déterministe ${ }^{98}$ de D. Bohm (né en 1917) va clarifier les paradoxes liés aux théorèmes sur l'inexistence des variables cachées. D'abord, il n'y a pas de «variables cachées » autres que des positions. Avant les interactions des dispositifs macroscopiques (ce qu'on appelle «mesures», mais à tort selon J. Bricmont), «il n’y a pas de valeur assignée par le système aux opérateurs variés tels que le moment, le spin, le moment angulaire, etc.» ${ }^{99}$. Sauf pour la position, toute «mesure» de quelque chose «est une interaction authentique entre le système et l'appareil » ${ }^{100}$. Ce qui veut dire que «la théorie de Bohm rend concrète et mathématiquement précise l'intuition de Bohr sur l'impossibilité de séparer le système de l'appareil » ${ }^{101}$.

J. Bricmont explique que l'observation de base de la théorie de Bohm est que «la fonction d'onde est une fonction définie sur l'espace de configuration et non, comme par exemple le champ électromagnétique, sur l'espace physique ${ }^{102}$. Soient les deux particules, s'il y a un potentiel externe agissant vers l'origine et correspondant à l'introduction d'un dispositif de mesure sur la première particule, alors la fonction d'onde évolue selon le potentiel de l'équation de Schrödinger, mais elle détermine les trajectoires des deux particules grâce à l'équation-guide. Et la trajectoire de la deuxième particule sera affectée par le potentiel même éloigné de l'origine: «la fonction d'onde met en corrélation des parties distantes de l'univers grâce à l'équation ${ }^{103}$ : une forme subtile de communication s'est donc établie entre les deux côtés de l'expérience.

\section{CONCLUSION}

Même si nous n'en avons pas conscience, non seulement nous vivons dans un monde quantique ${ }^{104}$, mais encore on peut dire aussi que «le monde est nonlocal ${ }^{105}$. Par le détour des instruments du laboratoire et des formulations mathématiques, les objets de la connaissance scientifique manifestent leur existence sinon visible du moins inférée. Il s'agit donc de se rallier à une Réalité inférée, déjà admise par Bachelard, et qui témoigne incontestablement en faveur du fait que «la» réalité préexistait à l'intervention des observateurs. De plus, on ne peut absolument pas dire qu'il y ait un «phénomène» avant qu'il ne soit observé:

98 What is the meaning of the wave function?, p. 10. Dans Contre la philosophie de la mécanique quantique, in R. Franck (éd.), Les sciences et la philosophie. Quatorze essais de rapprochement, Paris, Vrin, 1995, pp. 132-175, J. Bricmont montre l'état du problème, p. 166: 1) soit on adopte des univers multiples; 2) soit on introduit les variables cachées de Bohm: les positions; 3) soit on adopte une théorie stochastique ou non linéaire. Pour Bell, les solutions 2 et 3 donnent une image précise.

100 Ibid.

101 Ibid.

102 Ibid.

103 Ibid.

104 Lochak, Diner, Fargue, L'objet quantique, p. 7.

105 Contre la philosophie de la mécanique quantique, in Les sciences et la philosophie, p. 145. 
constatation de J. A. Wheeler (né en 1911) ${ }^{106}$, valable aussi bien en mécanique classique qu'en mécanique quantique où, comme nous l'avons vu, elle prend un sens tout particulier. Lorsqu'on parle de «réalité » ou de «matière», il faut pouvoir préciser le niveau auquel on place les entités qui y correspondent. Dénuée de sa masse et se déplaçant à la vitesse de la lumière ou, inversement, énergie refroidie et devenue inerte de sorte que sa masse puisse être mesurée, seule la matière scientifique est quelque chose de déterminé. L'observation scientifique de la réalité préexistant à l'intervention des dispositifs comme à celle de la pensée conceptuelle est toujours une observation armée, médiatisée conceptuellement ou techniquement.

\section{Groupe d'Etudes et de Recherches Epistémologiques Paris}

\section{RÉFÉRENCES}

G. Bachelard, Le nouvel esprit scientifique, Paris, PUF, 1946 (1934).

J.-L. Basdevant \& J. Dalibard, Physique quantique, Paris, Ellipses, AUPELF/UREF, 1998.

J. S. Bell, Speakable and Unspeakable in Quantum Mechanics, Cambridge, Cambridge University Press, 1993.

H. Bergson, Euvres, Textes annotés par A. Robinet, Introduction par H. Gouhier, Paris, PUF, 1970 (1959).

D. Bohm, A suggested interpretation of the quantum theory in terms of «hidden variables», Parts 1 and 2, in «Phys. Rev.», 1952, nº 89, pp. 66-193.

N. Bohr, Can quantum mechanical description of reality be considered complete?, in «Phys. Rev.», 1935, nº 48, pp. 696-702.

F. Brentano, Psychologie du point de vue empirique (1874-1911), traduit par M. de Gandillac, Paris, Aubier, 1944.

J. Bricmont, Contre la philosophie de la mécanique quantique, in R. Franck (éd.), Les sciences et la philosophie. Quatorze essais de rapprochement, Paris, Vrin, 1995.

J. Bricmont, What is the meaning of the wave function?, first published in J.-M. Frere, M. Henneaux, A. Sevrin \& Ph. Spindel (eds), Fundamental interactions: from symmetries to black holes, Conference held on the occasion of the «Eméritat» of François Englert, Brussels, Université Libre de Bruxelles, 1999, pp. 53-67.

J. Bricmont, La non-localité et la théorie de Bohm, Académie des sciences morales et politiques, 2001, pp. 1-12, http://www.asmp.fr.

J. Bricmont, Bayes, Boltzmann and Bohm: Probabilities in Physics, in J. Bricmont, D. Durr, M. C. Galavotti, F. Petruccione \& N. Zanghi, (eds), Chance in Physics, Heidelberg, Springer Verlag, 2001.

J. Bricmont, Postmodernism and its problems with science, http://dogma.free.fr, Lecture given at Helsinki at the invitation of the Finnish Mathematical Society, 2002

J. Bricmont \& A. Sokal, Physical Realism from a Physical Perspective, Bielefeld, Juin 2001, Welt und Wissen - Monde et savoir - World and Knowledge (à paraître).

J. Bricmont, A. Kremer-Marietti, D. Lecourt, F. Nègre, T. Simonelli \& A. Spire, La science nous ditelle la vérité?, dossier in «Res Publica», juin 2001, n 25.

106 Je me réfère à la proposition établie par J.A. Wheeler cité par J. Bricmont, What is the meaning of the wave function?, note 5 . 
E. Cassirer, La philosophie des formes symboliques, t. III, La phénoménologie de la connaissance, traduit par O. Hansen-Love, J. Lacoste \& C. Frontyl, Paris, Minuit, 1972 (1929).

A. Einstein, B. Podolsky \& N Rosen, Can quantum mechanical description of reality be considered complete?, in «Phys. Rev.», 1935, n 47, pp. 777-780.

P. Feyerabend, Contre la méthode. Esquisse d'une théorie anarchiste de la connaissance, traduit par B. Jurdant \& A. Schlumberger, Paris, Seuil, 1979 (1975).

S. Goldstein, Quantum theory without observers, in «Physics Today», March 1998, pp. 42-46, April 1998, pp. 38-42. «Physics Today», «Letters », February, 1999.

M. Heidegger, Sein und Zeit, traduction française de F. Vezin, Etre et temps, Paris, Gallimard, 1986 (1927).

W. Heisenberg, Philosophie. Le manuscrit de 1942, Paris, Seuil, 1998.

W. Heisenberg, La nature dans la physique contemporaine, traduit de l'allemand par U. Karvelis \& A. E. Leroy, Paris, Gallimard, 1962 (1955); nouv. éd. introduite par C. Chevalley, Paris, Gallimard, Folio-Essais, 2000.

P. Jacob, Working paper 99-6, http://www.isc.cnrs.fr

K. Jaspers, Psychologie der Weltanschauungen, Heidelberg, Muenchen, Springer Verlag, 1919.

I. Kant, Critique du jugement, traduit par Gibelin, Paris, Vrin, 1960 (1790).

I. Kant, Critique de la raison pure, traduit par A. Tremesaygues \& B. Pacaud, Paris, PUF, 1975 (1781, 1787).

A. Kremer-Marietti, Jaspers et la scission de l'être, Paris, Seghers, 1967, 1974; L'Harmattan, 2002.

A. Kremer-Marietti, Dilthey et l' anthropologie historique, Paris, Seghers, 1971.

A. Kremer-Marietti, La raison créatrice. Moderne ou postmoderne, Paris, Kimé, 1996.

A. Kremer-Marietti (dir.), Sociologie de la science, Sprimont (Belgique), Pierre Mardaga, 1998.

A. Kremer-Marietti, Philosophie des sciences de la nature, Paris, PUF, 1999.

A. Kremer-Marietti (dir.), Ethique et épistémologie autour du livre Impostures intellectuelles, de Sokal et Bricmont, Paris, L'Harmattan, 2001.

T. S. Kuhn, La structure des révolutions scientifiques, traduit par L. Meyer, Paris, Flammarion, Collection «Champs », 1983 (1962, 1970).

G. Lochak, S. Diner, \& D. Fargue, L'objet quantique. Comment l' esprit vient aux atomes, Paris, Flammarion, Collection Champs Flammarion, 1989.

E. Mach, La Mécanique, traduit par Bertrand, Paris, Hermann, 1904 (Titre orignal Die Mechanik in ihrer Entwicklung historisch-kritisch dargestellt, 1883).

E. Mach, L'analyse des sensations, traduit par F. Eggers \& J.-M. Monnoyer, Nîmes, J. Chambon, 1996 (1882).

E. Mach, The Analysis of Sensations, Translation by C. M. Williams \& Sydney Waterlow, Dover Edition, 1959 (1897).

G. Marcel, Journal métaphysique, Paris, Gallimard, 1927.

D. Pestre, Introduction, in La science et la guerre, dossier de « La Recherche », avril-juin 2002, $n^{\circ} 7$, hors série.

J. Piaget, Introduction à l'épistémologie génétique, Paris, PUF, 1974 (1950).

J.-Ch. Sacchi, Sur le développement des théories scientifiques. De l'aporie de l'incommensurabilité à la dimension pragmatique de la découverte, Paris, L'Harmattan, 1999.

B. Spinoza, Euvres, t. I, traduit et annoté par C. Appuhn, Paris, Garnier, 1964.

H. Umezawa \& G. Vitiello, Quantum Mechanics, Napoli, Bibliopolis, 1985.

J.A. Wheeler, in Mathematical Foundations of Quantum Mechanics, A. R. Marlow (ed.), New York, Academic Press, 1978. 